\title{
«lch möchte EMH als Medienhaus positionieren»
}

\author{
Interview: Bruno Kesseli
}

Dr. med. et lic. phil., Chefredaktor

\begin{abstract}
Vor rund einem Jahr hat Hans Kurt das Amt des Verwaltungsratspräsidenten des Schweizerischen Ärzteverlags EMH übernommen, zu dessen Kernprodukten auch die Schweizerische Ärztezeitung zählt. Eine Zwischenbilanz in Interviewform.
\end{abstract}

\begin{abstract}
Hans Kurt, Sie haben im Juni vergangenen Jahres Ludwig T. Heuss als Verwaltungsratspräsident des Schweizerischen Ärzteverlags EMH abgelöst. Zuvor waren Sie bereits während mehrerer Jahre als Vertreter der FMH Mitglied des Verwaltungsrats. Was hat Sie dazu motiviert, den Präsidentenstab von Ihrem Vorgänger zu übernehmen?
\end{abstract}

Ich habe den EMH-Verlag in meiner Zeit im Verwaltungsrat als spannendes und sich in Entwicklung befindendes Unternehmen erlebt. Sowohl im Verwaltungsrat als auch in der Geschäftsleitung arbeiten interessierte und engagierte Leute aus Medizin und Verlagswesen zusammen. Die Stimmung ist sehr freundschaftlich, wohlwollend. Ich mag diese Art von Zusammenarbeit, sie macht kreativ und bringt viel gegenseitige Wertschätzung mit sich.

Sie sind nun seit knapp einem Jahr VR-Präsident. Was hat sich durch das neue Amt für Sie verändert?

Als Präsident fühle ich mich vermehrt in der Verantwortung: zum einen gegenüber der FMH, deren gewählter Vertreter ich schliesslich bin, zum anderenund dies ganz besonders - gegenüber EMH als Unternehmen.

\begin{abstract}
Können Sie dies etwas ausführen?
$\mathrm{EMH}$ ist ein angesehenes Unternehmen mit 20 Angestellten und soll sich auch in Zukunft entwickeln können. Zusammen mit dem Geschäftsführer und den Verantwortlichen der Geschäftsleitung versuche ich, die Kontakte zur FMH, aber auch zu anderen Partnern wie etwa dem Westschweizer Verlag Médecine et Hygiène zu intensivieren. Ich möchte EMH als Medienhaus positionieren, das Dienstleistungen aus der Medienwelt allen Ärzten zur Verfügung stellt, das heisst vermehrt auf die Bedürfnisse der kantonalen Ärztegesellschaften, der Fachgesellschaften oder anderer Organisationen aus der Medizin eingeht.
\end{abstract}

«Der EMH-Verlag ist nicht einfach einer von vielen im medizinischen Verlagsmarkt, sondern die Stimme der schweizerischen Ärzteschaft."
EMH ist in verschiedener Hinsicht ein spezieller Verlag. Im Gespräch mit Berufskolleginnen und -kollegen stellt man immer wieder fest, dass die Vorstellungen über die Unternehmensform bezie- hungsweise die dahinterstehende "Idee $E M H$ » sehr vage sind. Könnten Sie hier als VR-Präsident etwas Aufklärungsarbeit leisten?

\section{Dr. med. Hans Kurt}

In Solothurn aufgewachsen. Studium der Medizin an den Universitäten Freiburg i.Ü. und Bern. Seit über 25 Jahren als Facharzt für Psychiatrie und Psychotherapie in einer grösseren interdisziplinären Gruppenpraxis in Solothurn tätig (www.gruppenpraxisweststadt.ch). Ausbildung in systemischer Therapie und Supervision. Verschiedene Verbands- und gesundheitspolitische Mandate: langjähriger Präsident und Vorstandsmitglied der Schweizerischen Gesellschaft für Psychiatrie und Psychotherapie SGPP \& FMPP, Präsident Aktionsbündnis psychische Gesundheit Schweiz. Vizepräsident Verwaltungsrat der Solothurner Spitäler AG bis 2014, Verwaltungsrat EMH Schweizerischer Ärzteverlag seit 2009, ab 2014 Mitglied Übergangsrat Universitäre Psychiatrische Dienste Bern.

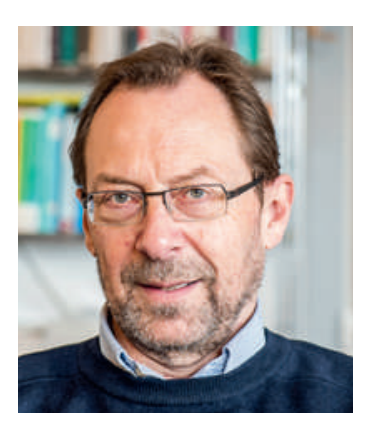


EMH zeichnet sich dadurch aus, dass sich vor 18 Jahren zwei ganz verschiedene Partner zusammengetan haben, um ein Unternehmen zu gründen. Zum einen war es die FMH, die bis heute die Aktienmehrheit hat, zum andern ein starker Partner aus dem Druck- und Verlagswesen, die Schwabe AG, die über eine lange Tradition im Bereich wissenschaftlicher Publikationen und qualitativ hochstehender Verlagsprodukte verfügt. EMH stellt aber ein eigenständiges Unternehmen dar und ist nicht einfach eine Unterorganisation der FMH. In diesem Sinn muss der Ärzteverlag auch eine eigene Unternehmenspolitik fahren können, nicht zuletzt, damit er sich im heftig umstrittenen Markt der medizinischen Publikationen an der Spitze behaupten kann.

\section{Inwiefern hebt sich EMH von anderen Medizin- verlagen $a b$ ?}

Der EMH-Verlag ist nicht einfach einer von vielen im medizinischen Verlagsmarkt, sondern die Stimme der schweizerischen Ärzteschaft, was manchmal gegenüber der Konkurrenz auch Schwierigkeiten machen kann. Dabei darf nicht vergessen werden, dass zwar die FMH die Aktienmehrheit hat und auch den Verwaltungsrat präsidiert, das Unternehmen aber von zwei gleichberechtigten Partnern getragen wird.

\section{«Schwierigkeiten gegenüber der Konkurrenz» - was ist damit gemeint?}

EMH verfolgt in Sachen Verlagspolitik eine strikte Unabhängigkeit gegenüber Inserenten und Geldgebern. Der Verlag will publikationsethische Richtlinien befolgen und hält weitere Werte hoch, nämlich ein sozialer Arbeitgeber zu sein und seine Produkte in der Schweiz herzustellen, und zwar wenn immer möglich nach ökologischen Kriterien.

"EMH verfolgt in Sachen Verlagspolitik eine strikte Unabhängigkeit gegenüber Inserenten und Geldgebern.»

\section{Entspricht ein Modell, wie Sie es eben skizziert haben, noch den Erfordernissen der Zeit? Warum braucht es EMH heute noch?}

Die Ärzteschaft und damit die FMH sind aus gesundheits- und standespolitischen Gründen auf die Herausgabe von eigenständigen Medienprodukten angewiesen. Dabei handelt es sich nicht nur um Zeitschriften, sondern vermehrt auch um Angebote aus der elektronischen Medienwelt. Da die FMH selbst kaum über Kompetenzen und Ressourcen im Verlags- und Druckwesen verfügt, braucht sie einen zuverlässigen Part- ner, der zum einen in der Fachwelt Ansehen geniesst und zum andern eine hochstehende Qualität garantieren kann.

\section{Die Zeiten haben sich seit der Gründung von EMH in verschiedener Hinsicht geändert - auch im Bereich der medizinischen Publizistik.}

EMH wird nächstes Jahr das zwanzigjährige Jubiläum feiern können. Art. 2 der Statuten von 1997 besagt, der Zweck der Aktiengesellschaft soll die Herausgabe von Medienerzeugnissen im Medizinalbereich sein. Daran hat sich bis heute nichts geändert. Aber die Form der Medienerzeugnisse und das Leseverhalten haben sich in den letzten Jahren gewandelt. Es braucht zwar weiterhin gut recherchierte, wissenschaftlich einwandfreie Artikel, aber gleichzeitig auch Kurzinformationen à la 20 Minuten, Diskussionen in Blogs und einen raschen, verlinkten Zugang auf ausgewählte Themen. Und der altehrwürdige Impact Factor bekommt Konkurrenz zum Beispiel durch «altmetrics» und «Google Scholar».

\section{«Der rasche Wandel in der Publizistik und} die rasanten Veränderungen in den Medien verlangen eine ständige Weiterentwicklung der bestehenden Angebote und die Offenheit für Neues.»

\section{Wo liegen die besonderen Stärken des Unternehmens und in welchen Bereichen sehen Sie die grössten Chancen für EMH?}

$\mathrm{Zu}$ den Stärken von EMH zählen sicher die schlanke Unternehmensstruktur und das grosse Mitarbeiterpotential. Alle Mitarbeiter und Mitarbeiterinnen der $\mathrm{EMH}$, die ich bis jetzt kennengelernt habe, sind bestens qualifiziert, mit Herzblut bei ihrer Arbeit und identifizieren sich stark mit dem Unternehmen. Des Weiteren nimmt der EMH-Verlag dank seiner hochwertigen Produkte in der medizinischen Publizistik der Schweiz eine Spitzenposition ein, was auch entsprechende Umfragen belegen. Dass EMH auf einer Kooperation zwischen der FMH und Schwabe basiert, gewährleistet ein hohes Ansehen des Verlags in der Öffentlichkeit.

\section{Wo orten Sie die grössten Herausforderungen und Risiken?}

Aus meiner persönlichen Sicht stehen wir in Zukunft vor zwei verschiedenen Herausforderungen: Der Übergang von Printmedien zu elektronischen Medien und die zukünftige Finanzierung unabhängiger Medien. EMH hat schon früh im Internet Fuss gefasst und sich für einen Open Access seiner Publikationen eingesetzt. Das Swiss Medical Weekly (www.smw.ch) trat als eine der ersten medizinischen Fachzeitschriften mit einer 


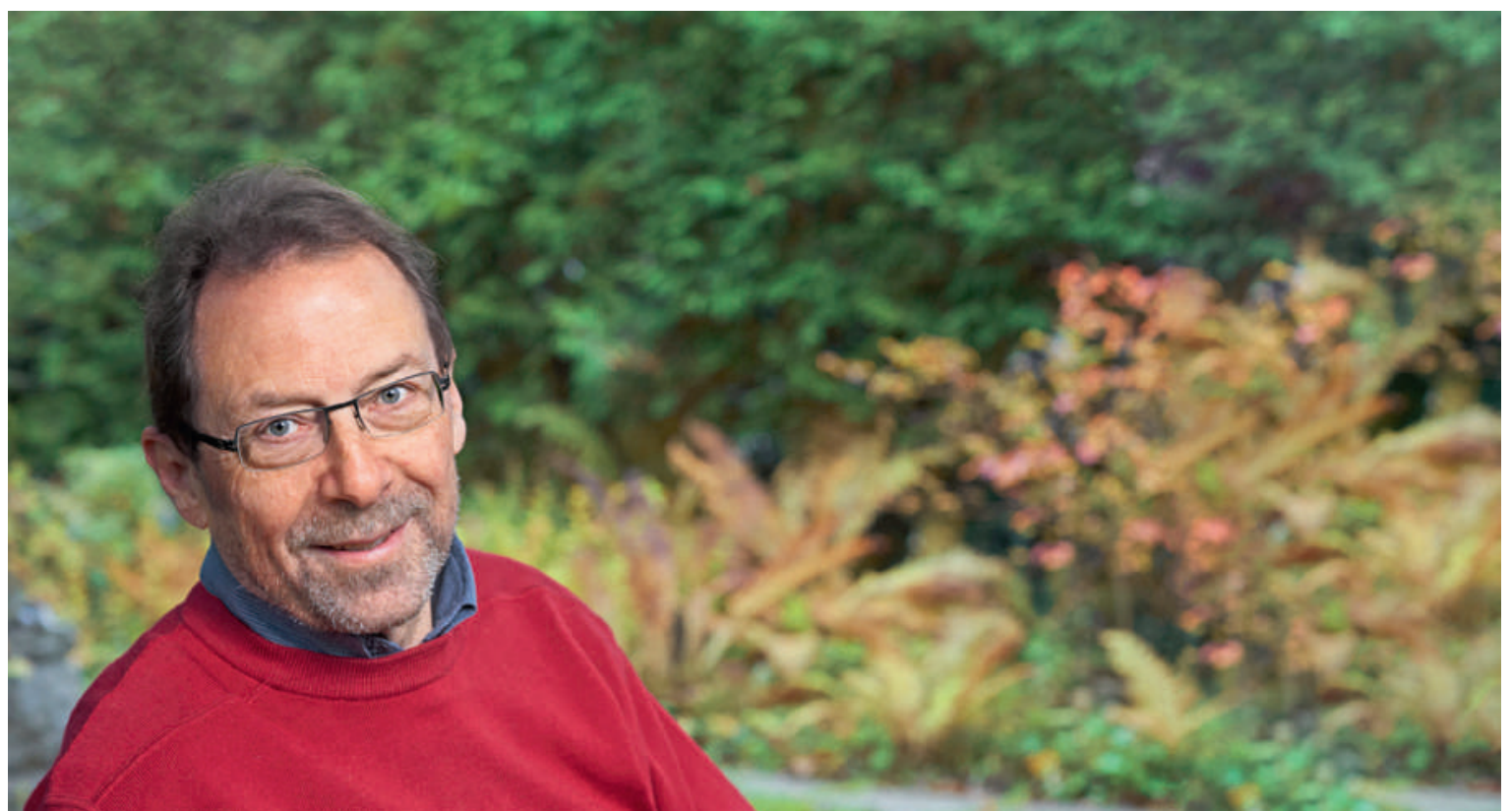

Fühlt sich als Präsident vermehrt in der Verantwortung: Hans Kurt.

eigenen Website auf. Gerade das SMW als Online-OpenAccess-Zeitschrift zeigt exemplarisch neue Möglichkeiten und Schwierigkeiten auf. Der rasche Wandel in der Publizistik und die rasanten Veränderungen in den Medien verlangen eine ständige Weiterentwicklung der bestehenden Angebote und die Offenheit für Neues. Dies alles geschieht in einem ökonomisch schwierigen Umfeld, in dem Sparen angesagt ist und die traditionellen Finanzierungsquellen drastisch zurückgehen.

\section{Wie lassen sich solche Entwicklungen finanzieren?} Schon jetzt muss EMH mit seinen Mitteln sehr haushälterisch umgehen und sich seine finanzielle Unabhängigkeit andauernd erarbeiten. Dabei unterliegt der Verlag dem gleichen Spardruck wie die FMH, die entsprechende Forderungen stellt. Die finanziellen Ressourcen werden trotz der kommenden Herausforderungen, die eigentlich einen Mehrbedarf nach sich ziehen würden, nicht grösser werden. Persönlich denke ich, dass in Zukunft qualitativ gute und attraktive Medienprodukte nicht mehr ohne Bezahlung in der einen oder anderen Form - Abonnemente oder anderes - möglich sein werden.

\section{Wie sehen Sie den künftigen Stellenwert der Inserate, aus denen sich die Zeitschriften von EMH bisher hauptsächlich finanzieren?}

Unabhängigkeit und hohe Qualität lassen sich in $\mathrm{Zu}$ kunft wohl nicht mehr im selben Ausmass über Inserate und Sponsoren decken, wie dies lange der Fall war. Vergessen wir nicht, dass die FMH über viele Jahre von den erwirtschafteten Überschüssen profitiert hat. Diese Zeiten sind mit dem schwindenden Inseratemarkt leider vorbei. Insofern bedeutet die hohe Abhängigkeit von Inseraten, insbesondere aus dem Bereich der pharmazeutischen Industrie, aber auch in Bezug auf den Stellenmarkt, in der Zukunft ein erhebliches Risiko für EMH. Ein dauerhaftes Engagement seitens der FMH-Mitglieder mit einem adäquaten Abonnement würde helfen, die jährlich wiederkehrenden Unsicherheiten abzufedern.

"Grundsätzlich wird sich EMH in den neuen Medien positionieren, aber mit qualitativ ansprechenden Produkten.»

\section{Welche Strategie hat EMH in Bezug auf die sogenann-} ten neuen Medien, die zwar nicht mehr so neu sind, sich aber rasant weiterentwickeln?

EMH ist bereits heute daran, verschiedene Schritte in der sogenannten neuen Medienwelt zu unternehmen. Alle unsere Printprodukte wurden in der Zwischenzeit so verändert und produziert, dass sie auch in elektronischer Form mit den entsprechenden Gadgets zur Verfügung stehen. Es ist aber durchaus notwendig, vermehrt im Internet Fuss zu fassen.

\section{Woran denken Sie?}

Zum Beispiel an Weiterbildungsangebote im Web. In Diskussion steht auch ein Web-Auftritt, der auf die breite Öffentlichkeit ausgerichtet ist, wobei zu berück- 
sichtigen wäre, dass die FMH als Hauptaktionär mit den entsprechenden Produkten und Informationen assoziiert würde. Beim Swiss Medical Weekly, das bereits jetzt eine Online-Zeitschrift ist, sehen wir aber, wie schwierig eine Finanzierung für solche Produkte ist. Auch hier müssen wir uns neue Wege für Finanzierungsmöglichkeiten überlegen. Grundsätzlich wird sich EMH in den neuen Medien positionieren, aber mit qualitativ ansprechenden Produkten und nicht einfach billigen, auf Effekt ausgerichteten Medienauftritten.

Die Meinungen über den zukünftigen Stellenwert von Printmedien, also im Fall von EMH der gedruckten Zeitschriften, gehen in der Fachwelt weit auseinander. Wie sehen Sie die Zukunft der EMH-Produkte in diesem Bereich?

Den Printmedien wird seit Jahren der Untergang prophezeit. Trotzdem gibt es einen grossen Leserkreis, der weiterhin die SÄZ oder das SMF gerne auf Papier liest. Es ist auch eine Altersfrage: Jüngere Ärzte werden zunehmend Online-Angebote wünschen, die ältere Ärzteschaft - und sie ist nicht unwesentlich - bevorzugt

\section{«Als Verwaltungsratspräsident möchte ich dieses Vertrauen aufbauen und günstige Bedingungen erarbeiten, damit sich der EMH- Verlag entwickeln kann.»}

die traditionelle Form der Zeitschriften. Eine Möglichkeit, den Übergang zu meistern, besteht darin, dass wir zunehmend die Redaktionen mit jungen Kollegen ergänzen, die eher wissen, welche Bedürfnisse zu berücksichtigen sind.

\section{Wo wollen Sie als VR-Präsident in den nächsten Jahren persönliche Akzente setzen? Welche Ziele liegen Ihnen besonders am Herzen?}

Eigentlich habe ich zwei Wünsche für die nächsten Jahre: Zum einen wünsche ich mir, dass seitens der Ärzteschaft das Vertrauen in ihren Ärzteverlag EMH wächst und sich Verwaltungsrat und Geschäftsleitung nicht immer wieder Vorurteilen und Kritiken gegenüber sehen, die ungerechtfertigt sind. Der Ärzteverlag hat es dringend nötig, nach den turbulenten Jahren, den verschiedenen finanziellen Überprüfungen und Analysen seine Arbeit wieder auf das eigentliche Geschäft konzentrieren zu können. Als Verwaltungsratspräsident möchte ich dieses Vertrauen aufbauen und günstige Bedingungen erarbeiten, damit sich der EMHVerlag entwickeln kann. Dazu gehört auch, dass wir dem Wohlergehen der Mitarbeiter und Mitarbeiterinnen genügend Beachtung schenken.

\section{Und der zweite Wunsch?}

Das zweite wichtige Anliegen für mich ist, dass die Medienprodukte von EMH, welcher Art sie auch immer sein mögen, unabhängig bleiben und einer ethischen Publizistik gehorchen. Die Meinungsvielfalt in der Ärzteschaft, aber auch in der Gesundheitspolitik soll gewahrt bleiben. Der EMH-Verlag will sich weder von der pharmazeutischen Industrie beeinflussen lassen, noch von Interessenvertretern, welcher Art auch immer.

\section{Zum Schluss: Macht Ihnen die Arbeit im zweifellos nicht einfachen Umfeld der medizinischen Fach- medien nach einem Jahr an der Verlagsspitze noch Freude? Bleiben Sie EMH noch eine Weile als VR-Präsi- dent erhalten?}

Ich finde die Arbeit bei EMH seit jeher interessant und nun als Präsident des Verwaltungsrates natürlich auch herausfordernd. Ich war ja selber früher bei Zeitschriften des EMH-Verlags engagiert, und habe erlebt, welchen Aufwand die Herstellung und Redaktion guter medizinischer Zeitschriften bedeutet. So wie mir früher das Schreiben Spass gemacht hat, macht mir heute die Arbeit bei EMH zusammen mit der FMH und den Verantwortlichen von Schwabe Spass. Das heisst, ich bleibe sicher noch!

Bildnachweise

Hans Kurt 